\title{
Z Research Square

\section{Effects of Encapsulated Cinnamaldehyde on Growth Performance, Intestinal Digestive and Absorptive Functions, Meat Quality and Gut Microbiota in Broiler Chickens}

Chongwu Yang ( $\square$ yangc315@myumanitoba.ca )

University of Manitoba https://orcid.org/0000-0002-2239-6494

Moussa Sory Diarra

Agriculture and Agri-Food Canada Guelph Research and Development Centre Janghan Choi

University of Manitoba

Argenis Rodas-Gonzalez

University of Manitoba

Dion Lepp

Agriculture and Agri-Food Canada Guelph Research and Development Centre

Shangxi Liu

University of Manitoba

Peng Lu

University of Manitoba

Marion Mogire

University of Manitoba

Qi Wang

Agriculture and Agri-Food Canada Guelph Research and Development Centre Joshua Gong

Agriculture and Agri-Food Canada Guelph Research and Development Centre Chengbo Yang

University of Manitoba

\section{Research}

Keywords: Broiler, Encapsulated essential oils, Gut health, Meat quality, Performance

Posted Date: March 2nd, 2021

DOI: https://doi.org/10.21203/rs.3.rs-253217/v1 
License: (c) (i) This work is licensed under a Creative Commons Attribution 4.0 International License. Read Full License 


\section{Abstract}

Background: Essential oils are potential antimicrobial alternatives and their applications in animal feeds are limited due to their fast absorption in upper gastrointestinal tract. This study investigated the effects of encapsulated cinnamaldehyde (CIN) at $50 \mathrm{ppm}$ or $100 \mathrm{ppm}$ on the growth performance, organ weights, meat quality, intestinal morphology, jejunal gene expression, nutrient digestibility, and ileal and cecal microbiota. A total of 320 male day-old broiler Cobb-500 chicks were randomly allocated to 4 treatments with 8 pens per treatment (10 birds per pen): 1) basal diet (negative control, NC); 2) basal diet supplemented with $30 \mathrm{ppm}$ avilamycin premix (positive control, PC); 3) basal diet with 50 ppm encapsulated CIN (EOL); 4) basal diet with 100 ppm encapsulated CIN (EOH).

Results: Despite birds fed EOH tended to increase $(P=0.05)$ meat $\mathrm{pH}$ at $24 \mathrm{~h}$, all $\mathrm{pH}$ values were normal. Similar to PC group, meat from birds fed EOL and EOH showed a reduced $(P<0.05)$ Warner-Bratzler force shear (WBFS) compared to the NC group. The highest villus to crypt ratios $(\mathrm{VH} / \mathrm{CD} ; P<0.05)$ were observed in broiler fed either EOL or EOH, with an average being $14.67 \%$ and $15.13 \%$ in the duodenum and $15.13 \%$ and $13.58 \%$ in the jejunum, respectively. For jejunal gene expressions, only six out of the 11 studied genes showed statistically differences among the dietary treatments. Gene expressions of cationic amino acid transporter 1 (CAT-1) and neutral amino acid transporter 1 (B0AT-1) were upregulated in EOH-fed birds compared to PC and NC-fed birds $(P<0.05)$, respectively; while expression of proliferating cell nuclear antigen (PCNA) were downregulated in EOL-fed birds when compared to NC birds $(P<0.05)$. Nonetheless, the expressions of cadherin $1(\mathrm{CDH}-1)$, zonula occludens 1 (ZO-1), and maltase-glucoamylase $(\mathrm{MG})$ were all upregulated $(P<0.05)$ in EOH-fed birds compared to PC-fed birds. The apparent ileal digestibility (AID) of dry matter, crude protein, crude fat and of all 18 tested amino acids increased in EOL-fed birds $(P<0.01)$. Additionally, relative abundances $(\%)$ of ilealProteobacteriadecreased, while ileal and cecal Lactobacillusincreasedin EOH-fed birds $(P<0.05)$.

Conclusions: Dietary encapsulated CINimproved meat quality and gut health by reducingmeat WBFS,increasing VH/CD in intestines, jejunal gene expressions, AID of nutrients and beneficiallyileal and cecal microbiota composition.

\section{Full Text}

This preprint is available for download as a PDF.

\section{Figures}




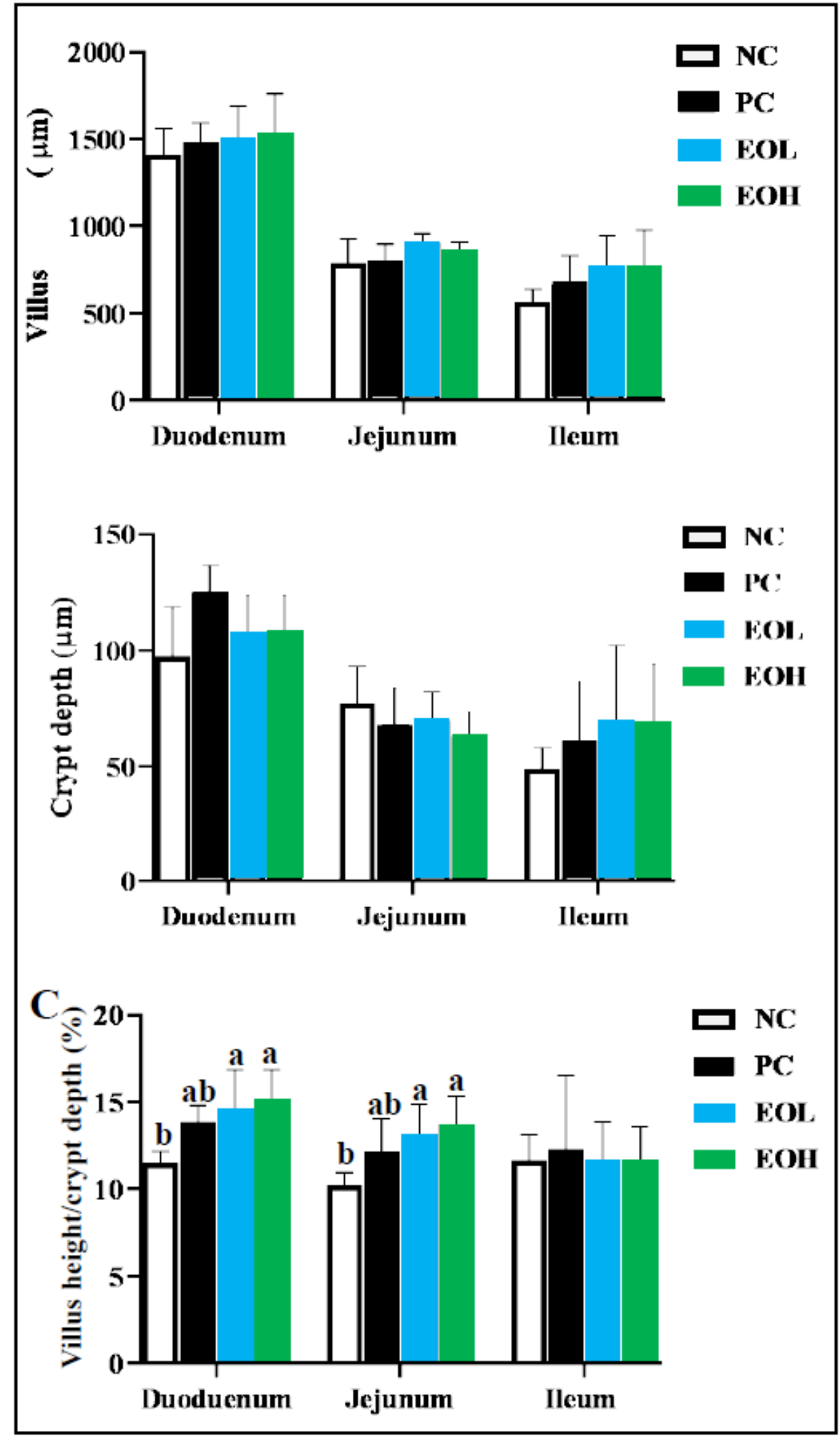

Figure 1

Effects of encapsulated cinnamaldehyde on villus height (A), crypt depth (B) and ratio of villus height and crypt depth (C) on duodenum, ileum, and jejunum in broilers. NC, negative control, birds fed with basal diet; PC, positive control, birds fed with 30 ppm avilamycin premix; EOL, birds fed 50 ppm encapsulated cinnamaldehyde; $\mathrm{EOH}$, birds fed 100 ppm encapsulated cinnamaldehyde. Significant differences are indicated by letters $(a, b)$. 
CDH-1

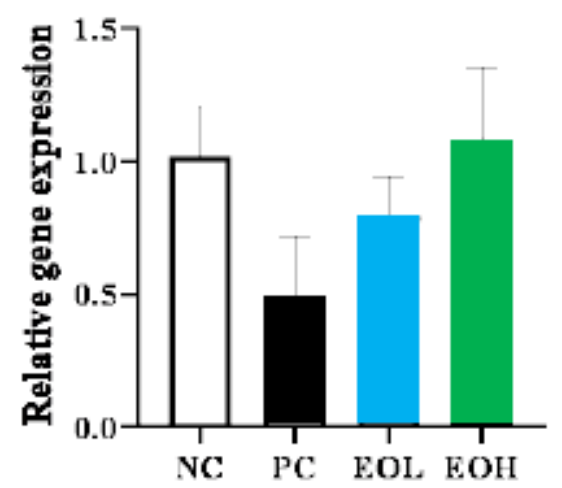

ZO-1

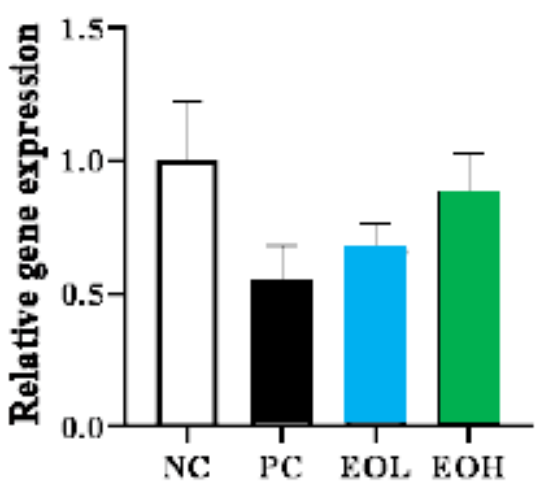

CAT-1

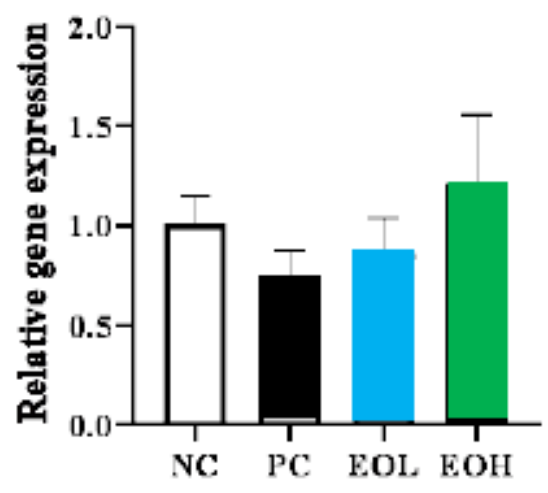

PCNA

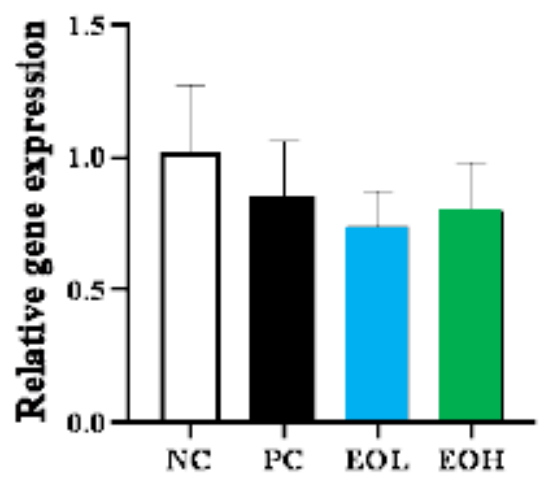

$\mathbf{B}^{0}$ AT-1

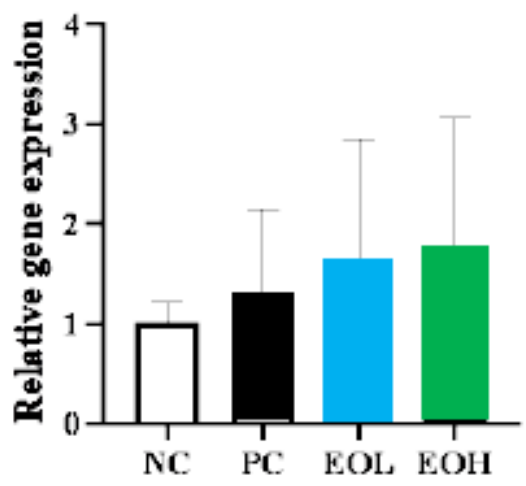

MG

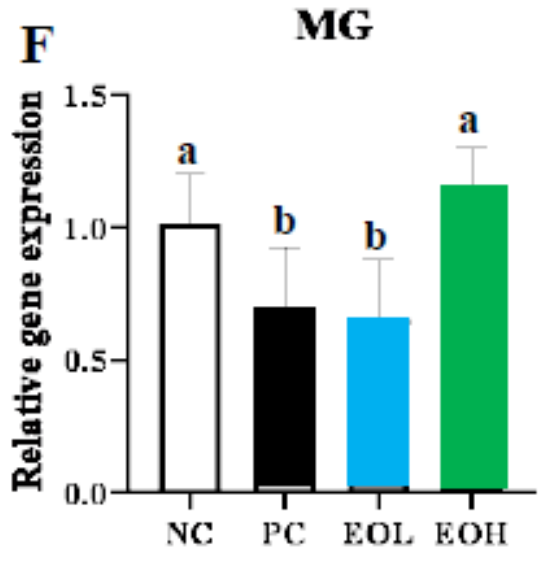

Figure 2

Effects of encapsulated cinnamaldehyde on jejunal gene expressions of CDH-1 (A), PCNA (B), ZO-1 (C), B0AT-1 (D), CAT-1 (E) and MG (F). NC, negative control, birds fed with basal diet; PC, positive control, birds fed with 30 ppm avilamycin premix; EOL, birds fed 50 ppm encapsulated cinnamaldehyde; EOH, birds fed 100 ppm encapsulated cinnamaldehyde; CDH-1, cadherin 1; PCNA, proliferating cell nuclear antigen; ZO-1, 

maltase-glucoamylase. Significant differences are indicated by letters $(a, b)$.

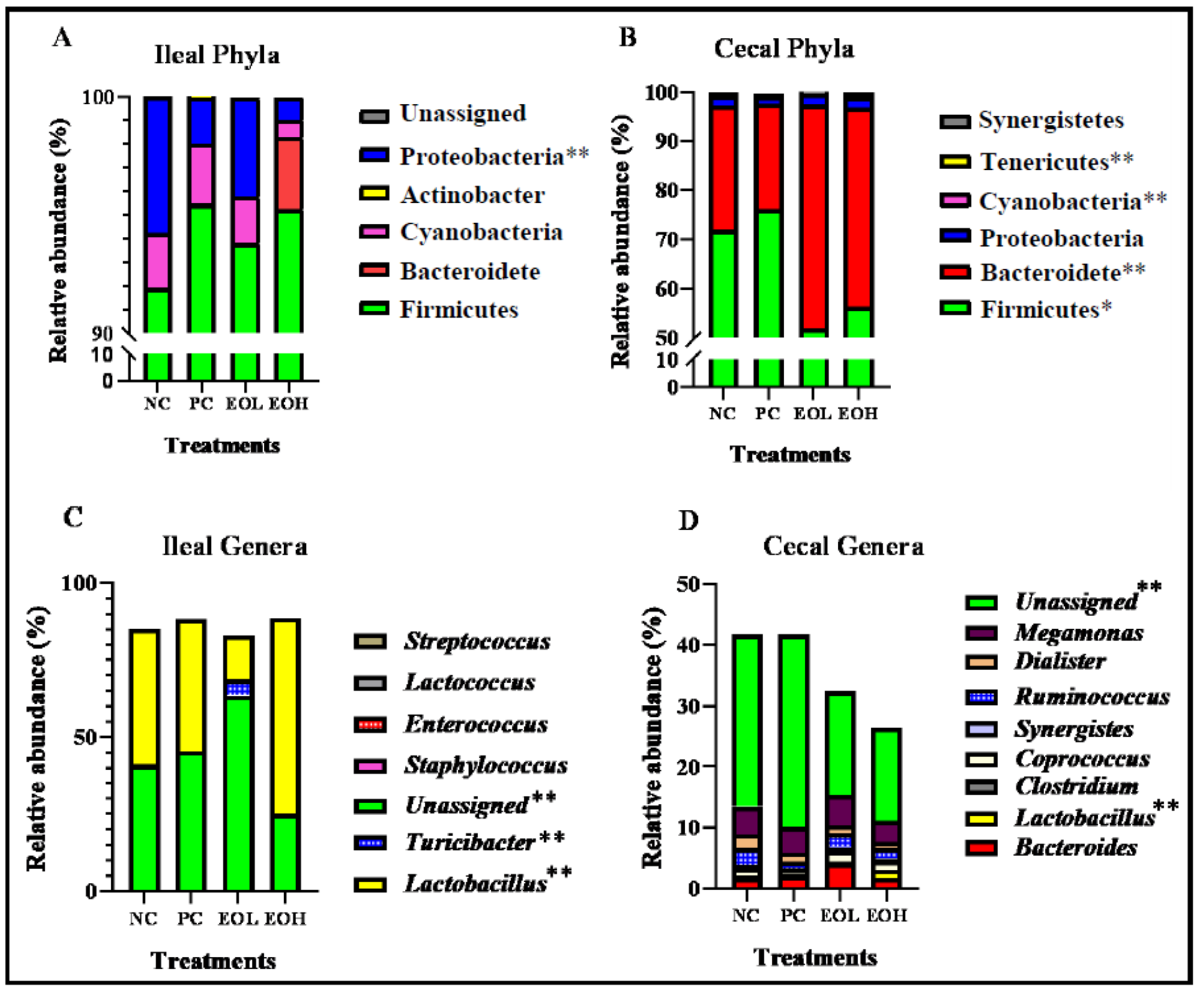

Figure 3

Relative abundance of ileal phyla (A) and major (>1\% relative abundance) genera (C) and cecal phyla (B) and major ( $>1 \%$ relative abundance) genera (D) in birds treated with $30 \mathrm{ppm}$ avilamycin or encapsulated cinnamaldehyde. NC, negative control, birds fed with basal diet; PC, positive control, birds fed with 30 ppm avilamycin premix; EOL, birds fed 50 ppm encapsulated cinnamaldehyde; $E O H$, birds fed 100 ppm encapsulated cinnamaldehyde. Asterisks indicate significant statistically differences (one asterisk means a significance level of 0.05 ; two asterisks mean a significance level of 0.01 ). 


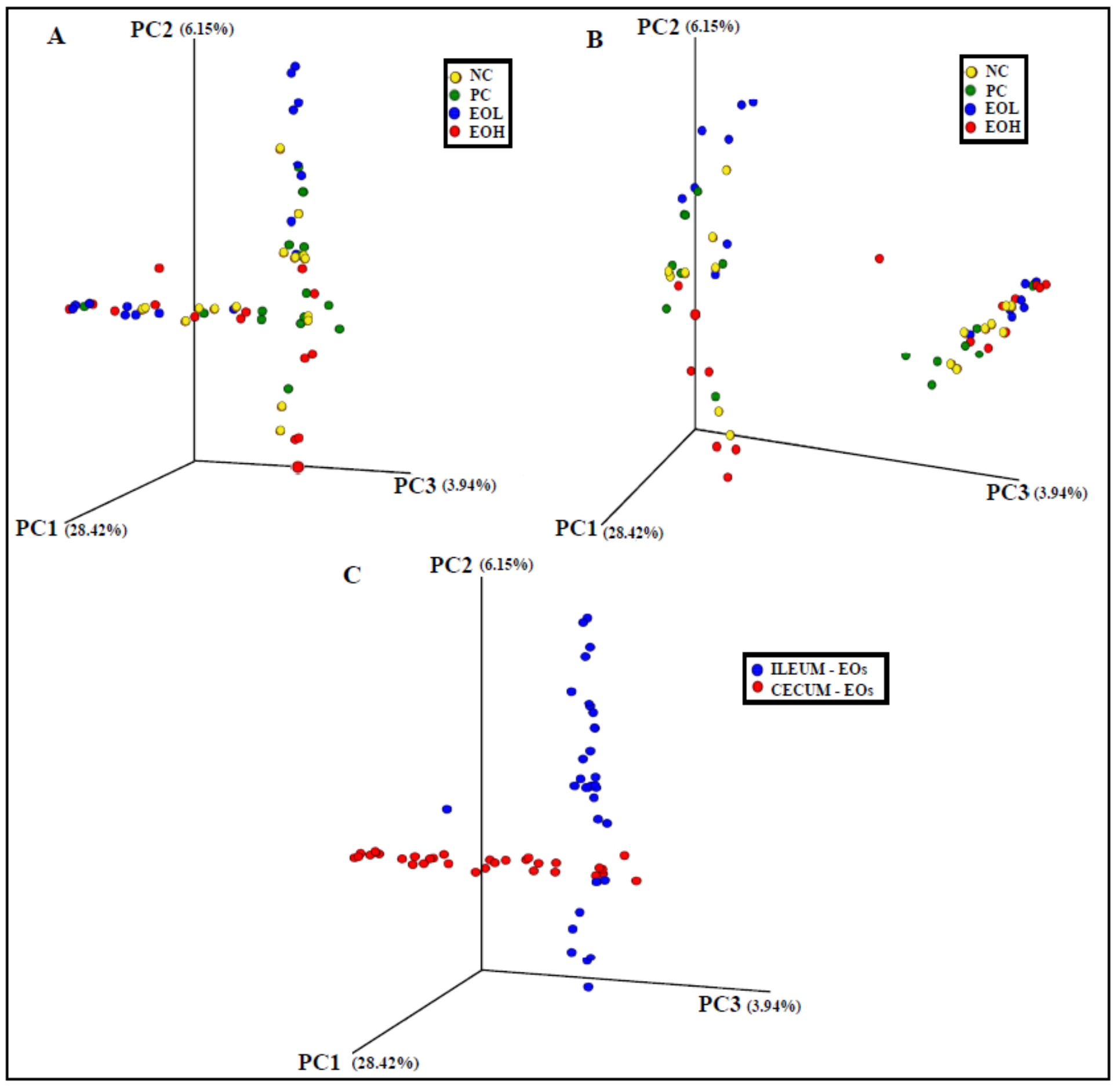

\section{Figure 4}

The 3D principal coordinate analysis (PCOA) graph shows the variation among distance matrixes (weighted UniFrac) of ileal (A) and cecal (B) microbiota alone or together (C) in birds treated with avilamycin premix or encapsulated cinnamaldehyde. Percentages shown are percentages of variation explained by the PC1 (28.42\%), PC2 (6.15\%), and PC3 (3.94\%). NC, negative control, birds fed with basal diet; PC, positive control, birds fed with 30 ppm avilamycin premix; EOL, birds fed 50 ppm encapsulated cinnamaldehyde; $\mathrm{EOH}$, birds fed 100 ppm encapsulated cinnamaldehyde. 